\title{
Reduced differentiation of emotion-associated bodily sensations in autism
}

Authors: Palser, E. R. ${ }^{1,2,3}$, Galvez-Pol, A. ${ }^{2,4}$, Palmer, C. E. ${ }^{2,5}$, Hannah, R. ${ }^{2,6}$, Fotopoulou, A. ${ }^{1}$, Pellicano, E. ${ }^{7,8}$, \& Kilner, J. M².

1 - Department of Clinical, Educational and Health Psychology, Psychology and Language Sciences, University College London, Gower Street, London WC1E 6BT, UK

2 - Department of Clinical and Movement Neurosciences, UCL Institute of Neurology, University College London, 33 Queen Square, London WC1N 3BG, UK

3 - UCSF Dyslexia Center, Department of Neurology, University of California San Francisco, 675 Nelson Rising Lane, San Francisco, CA, 94158, USA

4 - Psychology Department, University of the Balearic Islands, 07122, Palma de Mallorca, Spain

5 - ABCD Coordinating Center, Center for Human Development (CHD), University of California San Diego, USA

6 - Department of Psychology, University of California, San Diego, CA 92093, USA

7 - Centre for Research into Autism and Education, UCL Institute of Education, University College London, London, UK

8 - Department of Educational Studies, Macquarie University, Sydney, NSW 2109, Australia

\section{Abstract}

Differences in understanding emotion in autism are well-documented, although far more research has considered how being autistic impacts an understanding of other people's emotions, compared to their own. In neurotypical adults and children, many emotions are associated with distinct bodily maps of experienced sensation, and the ability to report these maps is significantly related to the awareness of interoceptive signals. Here, in 100 children who either carry a clinical diagnosis of autism $(n=45)$ or who have no history of autism $(n=55)$, we investigated potential differences in differentiation across autistic children's bodily maps of emotion, as well as how such differentiation relates to the processing of interoceptive signals. As such, we measured objective interoceptive performance using the heartbeat counting task, and participants' subjective experience of interoceptive signals using the child version of the Body Perception Questionnaire. We found less differentiation in the bodily maps of emotion in autistic children, but no association with either objective or subjective interoceptive processing. These findings suggest that, in addition to previously reported differences in detecting others' 
emotional states, autistic children have a less differentiated bodily experience of emotion. This does not, however, relate to differences in interoceptive perception as measured here.

Key words: Emotion; autism; interoception 


\section{Introduction}

Emotions have long been considered a fundamental part of the human experience. Evolutionary approaches emphasize the adaptive and innate role of many emotions, which help fine-tune behavioral responses allowing the individual to better match the challenges of their environment (Damasio, 1994; Levenson, 2003). Crucially, emotions are thought to represent a state of action readiness that involves the whole body. Being able to differentiate between many different emotional states is associated with a multitude of positive psychological outcomes, including good emotion regulation abilities (Barrett, Gross, Christensen, \& Benvenuto, 2001) and social skill (Pond et al., 2012). Similarly, the capacity to differentiate only a few different emotional states is linked to affective difficulties, including anxiety, depression, and self-injurious behavior (Kashdan, \& Farmer, 2014; Zaki, Coifman, Rafaeli, Berenson, \& Downey, 2013), all of which occur more frequently in autistic people (Gadow, DeVincent, Pomeroy, \& Azizian, 2004; Kim, Szatmari, Bryson, Streiner, \& Wilson, 2000; Muris, Sterrneman, Merchelbach, Holdrinet, \& Meesters, 1998; Simonoff et al., 2008; Soke et al., 2016; Sukhodolsky et al., 2008; Weisbrot, Gadow, DeVincent, \& Pomeroy, 2005).

Emotion difficulties have long been considered a component of the autism phenotype. Affective differences were included in the primary descriptions by Kanner (1943) and the assessment of emotional understanding and responding is still included in leading assessment tools, including the Autism Diagnostic Interview-Revised (ADI-R) (Rutter, Le Couteur, \& Lord, 2003) and Autism Diagnostic Observation Schedule $-2^{\text {nd }}$ edition (ADOS-2; Lord et al., 2012). While much research has been conducted on autistic people's ability to perceive and label others' emotional expressions, there is evidence to suggest that the experience of one's own emotions is also implicated. Approximately half of autistic people studied show signs of alexithymia, a difficulty identifying and describing emotional experiences (Griffin, Lombardo, \& Auyeung, 2016; Hill, Berthoz, \& Frith, 2004). When autistic adolescents are asked to respond to emotion-inducing photographs, they report a reduced range of emotional experiences and sort emotion words into fewer categories, suggesting they experience less differentiation between them (Erbas, Cuelemans, Boonen, Noens, \& Kuppens, 2013). Negative emotions may be disproportionately impacted, as autistic children have been found to particularly struggle with differentiating between sadness and fear (Rieffe, Terwogt, \& Kotronopoulou, 2007).

Central to many theories of emotion has been the role of afferent information about the internal bodily milieu. For example, James (1884) initially proposed that it is physiological sensations 
that give rise to subjective emotional states. There is a body of evidence demonstrating that those with greater access to their interoceptive states experience emotions more intensely (Barrett, Quigley, Bliss-Moreau, \& Aronson, 2004; Pollatos, Herbert, Matthias, \& Schandry, 2007; Wiens, Mezzacappa, \& Katkin, 2000; Zaki, Davies, \& Ochsner, 2012, although see Steptoe \& Vögele, 1992; Werner, Jung, Dunschek, \& Schandry, 2009; Werner, Kerschreiter, Kindermann, \& Duschek, 2013). Autism is commonly associated with difficulties in understanding and conveying emotions in the self and how they relate to internal states (Samson, Hardan, Podell, Phillips, \& Gross, 2015). While not always replicated (for example, see Nicholson et al., 2018; Shah, Hall, Catmur, \& Bird, 2016; \& Schauder, Mash, Bryant, \& Cascio, 2015 for null findings), there is evidence of altered interoception in both autistic children (Palser, Fotopoulou, Pellicano, \& Kilner, 2018) and adults (Garfinkel et al., 2016; Mul, Stagg, Herbelin, \& Aspell, 2018), as well as a significant link between these differences and affective symptomology, including anxiety and core diagnostic socio-emotional features (Garfinkel et al., 2016; Palser, Fotopoulou, Pellicano, \& Kilner, 2019).

The detection of bodily sensations is therefore considered key to emotional experience. A novel subjective report measure - the emBODY task - has recently been developed allowing researchers to ask participants which regions of the body are associated with the experience of emotion states (Nummenmaa, Glerean, Hari, \& Hietanen, 2014). This task requires participants to illustrate on an outline of the body the areas in which they experience a change in sensation during a particular emotional state. Using this method, several emotions were found to be associated with distinct self-reported bodily sensation maps in adults (Nummenmaa et al., 2014). The maps were also found comparable across Western European and Asian samples, suggesting they are robust to cultural and linguistic differences.

Bodily sensation maps of emotion have now been found to mature linearly over child development, with anger, happiness, surprise and fear showing a similar trajectory of early development and disgust and sadness showing later development, reaching full maturity in early adulthood (Hietanen, Glerean, Hari, \& Nummenmaa, 2016). The maturation of emotion-related bodily sensation maps in childhood broadly parallels increasing use of emotion words when describing events (Widen \& Russell, 2010), suggesting that the development of an awareness of emotion-related bodily sensations may promote children's ongoing emotional development. Interoceptive accuracy has been found to correlate positively with the magnitude of activation in emotion-related areas of the bodily sensation maps (Jung, Ryu, Lee, Wallraven, \& Chae, 2017). Thus, a greater ability to detect interoceptive signals is associated with more specific 
topographical changes during emotional states. Interoceptive sensibility, by contrast, refers to an individuals' subjective experience of interoceptive sensations (Garfinkel \& Critchley, 2013; Garfinkel, Seth, Barrett, Suzuki, \& Critchley, 2015), and heightened interoceptive sensibility has been found to co-occur with greater anxiety and alexithymia, including in autistic people (Ernst et al., 2013; Longarzo et al., 2015; Palser, Fotopoulou et al., 2018; Palser, Palmer et al., 2018). Differences in interoceptive processing in autism might then in turn relate to differences in the bodily experience of emotion.

Here, we used the emBODY task (Nummenmaa et al., 2014) to visualize the bodily emotion maps of autistic children and examine for the first time bodily emotion differentiation in this population. Children and adolescents with existing clinical diagnoses of an autism spectrum condition, as well as a comparison group of children and adolescents without a history of autism, completed the emBODY task and measures of interoceptive accuracy, sensibility, and alexithymia. A large sample of adults from the general population also completed the emBODY task to independently derive emotion-related regions of interest within the body maps. We hypothesized that there would be significant differences between autistic and non-autistic children in differentiation between emotions. Further, we predicted that this would be positively related to interoceptive accuracy and negatively related to interoceptive sensibility. 


\section{Methods}

Participants

\section{Child sample}

A total of 100 children and adolescents between 6 and 19 years of age were recruited from community contacts in London and the South East of England. Of the sample, $41 \%$ of participants were reported by parents to be White, $11 \%$ Asian, $8 \%$ Black, $12 \%$ Mixed, $5 \%$ of other ethnicity, and $22 \%$ declined to provide their ethnicity. Parents also reported the age at which they left full-time education, a widely used proxy for socioeconomic status (SES; Liberatos, Link, \& Kelsey, 1987), which was used to derive the number of years of education received beyond 16 years of age $(M=5.33, S D=3.89 \text {, range }=-1-17)^{1}$. One participant scored below 60 on our measure of IQ (standardized composite score; Wechsler Abbreviated Scales of Intelligence - second edition, WASI-II; Wechsler \& Hsiao-pin, 2011) and thus was excluded from analysis, leaving a final sample of 99 participants. Fifty-five participants were reported by parents to be neurotypical, with no ongoing or historic neurological or psychiatric conditions. The remaining participants had reportedly received an independent primary clinical diagnosis of an autism spectrum disorder, according to DSM-IV and ICD-10 criteria (DSM-IV-TR, 2000; ICD10, 1993). Additional co-occurring psychiatric and neurodevelopmental diagnoses reported included attention-deficit/hyperactivity disorder $(n=11)$, dyslexia $(n=9)$, dyspraxia $(n=3)$, anxiety disorder $(n=2)$, obsessive compulsive disorder $(n=1)$, Tourette's syndrome $(n=1)$ and Irlen syndrome $(n=1)$.

Ethical approval was granted by University College London institutional Ethics Board (Approval number: FPS 456) and all procedures were conducted in accordance with the Declaration of Helsinki. Parents of all children provided written informed consent for them to take part and the children also gave their assent.

To quantify autistic features, we used the Autism Diagnostic Observation Schedule $-2^{\text {nd }}$ edition (ADOS-2; Lord et al., 2012), and the parent-report Social Communication Questionnaire (SCQ; Rutter, Bailey, \& Lord, 2003). The ADOS-2 was only completed by children in the autistic sample, but both autistic and non-autistic children's parents were invited to complete the SCQ. SCQ data were missing from eight autistic children due to non-completion by parents and one

\footnotetext{
${ }^{1}$ One parent reported leaving full-time education at the age of 15 years, and so received a score of -1 when number of years of post-16 education was calculated. The parents of 29 participants declined to provide their age on leaving education.
} 
autistic child did not complete the ADOS-2 due to time constraints. With the exception of two children in the autistic sample, all met criteria for an autism spectrum disorder on at least one of these measures. We decided to retain the two participants who did not reach instrument criteria in the sample, given their existing clinical diagnoses of autism².

Table 1 shows participant characteristics. Of the non-autistic participants, 32 were female and 23 were male. Of the autistic participants, 9 were female and 35 were male, reflecting the gender disparity in the diagnosis of autism, which is approximately four males to every female diagnosed (Werling \& Geschwind, 2013). There were no significant group differences in age [U $=1231.000, p=0.884]$ or IQ $[t(89.562)=1.652, p=0.102]$. There was a significant difference in $\operatorname{sex}\left[X^{2}(1)=14.340, p<0.001\right]$; we therefore controlled for this difference in subsequent analyses.

Table 1: Characteristics of autistic and non-autistic participants in the sample. For sex, the ratio of males to females is given. For all other variables, the range, mean and standard deviation of scores is given, respectively.

\begin{tabular}{|l|c|c|}
\hline Characteristic & $\begin{array}{c}\text { Autistic participants } \\
(\mathbf{n = 5 5})\end{array}$ & $\begin{array}{c}\text { Non-autistic participants } \\
(\mathbf{n}=44)\end{array}$ \\
\hline Sex (m:f) & $35: 9$ & $23: 32$ \\
\hline Age & $7-19,13.27(2.75)$ & $6-18,13.18(3.39)$ \\
\hline $\mathrm{FSIQ}^{\mathrm{a}}$ & $64-133,98.71(16.56)$ & $75-138,104.09(15.55)$ \\
\hline $\mathrm{PIQ}^{\mathrm{a}}$ & $52-145,99.41(20.17)$ & $71-139,101.87(14.67)$ \\
\hline $\mathrm{VIQ}^{\mathrm{a}}$ & $67-135,98.80(15.39)$ & $70-144,105.07(16.62)$ \\
\hline $\mathrm{SCQ}^{\mathrm{c}}($ autistic $\mathrm{n}=37, \mathrm{non}-$ & $8-41,21.92(6.74)$ & $0-10,2.96(3.17)$ \\
autistic $\mathrm{n}=24)$ & & $\mathrm{n} / \mathrm{a}$ \\
\hline ADOS CSS & & \\
\hline
\end{tabular}

Notes: Age reflects chronological age and is given in years. ${ }^{a} / Q$ characteristics were measured using standardized composite scores on the Wechsler Abbreviated Scales of Intelligencesecond edition (WASI-II; Wechsler \& Hsiao-pin, 2011), n=99. FSIQ denotes full-scale IQ, PIQ denotes perceptual IQ and VIQ denotes verbal IQ. ${ }^{b} S C Q$ denotes scores on the Social Communication Questionnaire (Rutter, Bailey, \& Lord, 2003), where higher scores denote a greater number of symptoms. ' Autism severity score refers to calibrated severity score (CSS) on the Autism Diagnostic Observation Schedule - second edition (ADOS-2; Lord et al., 2012), revised algorithm (minimum score $=1$, maximum score $=10$ ) (Gotham, Risi, Pickles, \& Lord, 2007; Gotham, Pickles, \& Lord, 2009). Again, higher scores reflect greater severity.

\footnotetext{
${ }^{2}$ In addition, removing these two participants' data from the analyses did not change the results.
} 


\section{Adult sample}

A total of 314 members of the general population were also recruited through opportunity sampling as part of a public engagement event. These data were collected to generate an independent sample in which emotion-associated regions of interest could be derived, for use in analysis of the data from the child sample. Ethical approval was granted by the local institutional Ethics Board (Approval number: 0330/003) and all procedures were conducted in accordance with the Declaration of Helsinki. All participants provided written informed consent. Twenty-two of these participants were missing demographic data, and were therefore excluded, leaving a final sample of 292 participants (female: $n=204$; male: $n=88$ ), who ranged in age from 16 to 65 years $(M=32.37, S D=13.36)$. Data on ethnicity and SES were not collected from these participants.

\section{Measures}

\section{Emotional embodiment}

All participants completed a pen-and-paper version of the emBODY emotion coloring task (Hietanen et al., 2016; Nummenmaa et al., 2014) (see Figure 1). This task requires participants to illustrate within an outline of a human body (height $=8.4 \mathrm{~cm}$ ) with a felt tip pen, where they feel sensation during the labelled emotion state. Memory-based reports of emotions were used, instead of actively inducing emotional states. These methods have previously been shown to produce comparable body maps (Nummenmaa et al., 2014). Consistent with previous studies (Hietanen et al., 2016; Nummenmaa et al., 2014), for each emotion, the body was presented twice, side by side. For the left-hand side body, participants were instructed with the following written guidelines: "For this body, please color where activity gets stronger or faster". For the right-hand side of the body, participants were instructed: "For this body, please color where activity gets weaker or slower". For the child sample, the instructions were also given verbally and verbal confirmation that the children had understood the task was obtained from all. A total of six emotion states were presented to participants, in a randomized order: anger, fear, disgust, happy, sadness, and surprise, in addition to the state of emotional neutrality, which was termed 'calm'. Therefore, there were 14 outlines to respond to in total. Participants were instructed to leave an outline uncolored if they felt no change in sensation for that emotion state. 
Body maps were digitized and then pre-processed using a MATLAB (version R2016b) pipeline that was developed in-house. First, all maps were co-registered to a standard template, and areas outside the boundaries of the body outline were masked, such that pixels outside of the body could be discarded. Specifically, following Hietanen et al. (2016), maps were aligned to a reference fixed image by implementing a metric registration using Mattes mutual information. The alignment of the data was visually inspected and small corrections were performed when necessary. Each activation and deactivation map contained a total of 53,164 pixels.

Subject-wise activation and deactivation maps were converted into two-dimensional (2-D) matrices where each cell represents a pixel by coding pixels containing ink in both the activation and deactivation maps as (+1). Pixels containing no ink were coded as (0). Intensity of sensation was not measured in the present study. A combined subject-wise activationdeactivation map was created by subtracting the deactivation matrix from the activation matrix.

Figure 1: Pen-and-paper version of the emBODY emotion coloring task. All participants colored the initially blank bodies (Panel A) where they felt activity increasing (left-hand body) or decreasing (right-hand body). Activation and deactivation maps were aligned to a common template and masked, such that data outside the body outlines were removed. Subject-wise maps were then digitized (Panel $B$ ) by encoding any pixels colored on the Activation and Deactivation map as (+1). Any uncolored pixels were encoded as (0). As such, the entire body was represented by 53,164 data points. Subject-wise combined activation-deactivation maps were then combined by subtracting the Deactivation map from the Activation map (Panel C). 

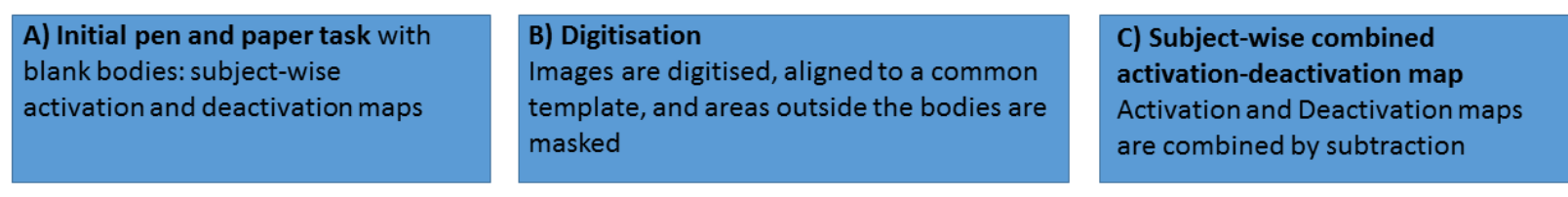

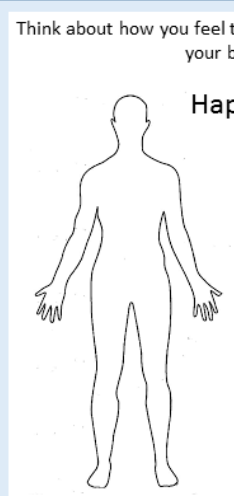

For this body, please colour the regions where activity gets stronger or faster

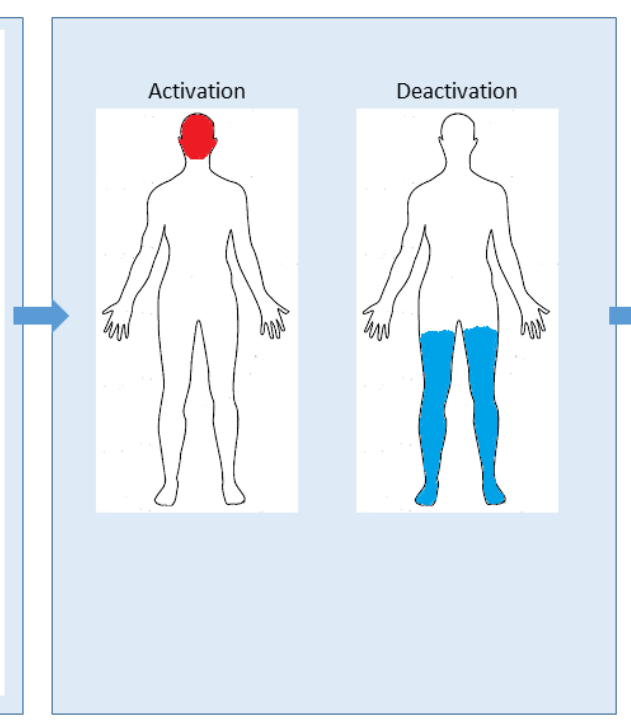



Bodily sensation maps were analysed using statistical non-parametric mapping using the SnPM plug-in (version 13) for the MATLAB toolbox SPM (version 12). SPM and SnPM were developed for the analysis of neuroimaging data. SnPM analysis is based on the principle of permutation testing. The null hypothesis of a permutation test is that the labels assigned to different testing conditions are arbitrary, and that the activation of a voxel will be comparable across conditions. To test this, a permutation test produces a distribution by either randomly assigning voxels to a condition label or randomly assigning a condition label to the voxels. This is repeated a nominal number of times; here we used 10,000 (SnPM default). For each iteration of the permutation, a $t$-statistic is generated and the $p$-value is determined as the proportion of $t$-statistics in the distribution that are greater or equal to the $t$-statistic of the observed data.

For example, with an alpha level of $p=0.05$, the null hypothesis is rejected if the observed $t$ statistic is located within the 95th percentile of the permutation distribution. Statistical nonparametric mapping is appropriate for use with non-parametric data, such as that which does not follow the normal distribution or is categorical. Here, we used statistical non-parametric mapping to analyse the pattern of activation observed not in voxels of the brain, but of the pixels in the bodily sensations maps. Regions of interest (ROIs) capable of dissociating between the different emotion conditions were determined in the independent general population sample 
using a within-subjects ANOVA in SnPM, with a cluster-forming threshold of $p=0.001$ and a family-wise error (FWE) correction of $p=0.05$.

Mean participant-wise activation was calculated for each emotion condition across the entire body map and in each of the ROIs. Emotion differentiation was operationalized in each participant as the variance (standard deviation) in mean activation over the whole body or ROI across the seven different emotion conditions.

\section{Interoceptive sensibility}

Interoceptive sensibility was measured in all participants using the modified version of the awareness subscale of the Body Perception Questionnaire (BPQ, Porges, 1993), adapted for children (Palser, Fotopoulou et al., 2018). Interoceptive sensibility data from the adult sample are not reported here but are analyzed elsewhere (Palser, Palmer et al., 2018). This child version is based on the 45-item questionnaire designed for adults (Porges, 1993). The modified version contains 39 items and participants are asked to indicate their awareness of the 39 bodily sensations described using a five-point scale ranging from 'Never' (1) to 'Always' (5).

Participants could either respond verbally or indicate their response on a chart on the table in front of them. This subjective measure of interoceptive sensibility denotes the participant's belief in his/her own interoceptive aptitude, irrespective of actual (objectively-determined) interoceptive accuracy. Interoceptive sensibility was operationalized as mean score on the modified version of the Awareness Scale of the BPQ (Porges, 1993; Palser, Fotopoulou et al., 2018). Reliability was very good for the autistic sample (Cronbach's alpha $=0.86$ ), and excellent for the non-autistic sample (Cronbach's alpha $=0.90)$.

\section{Interoceptive accuracy}

Interoceptive accuracy was measured in the child sample but not the adult sample. This was gauged by the participants' ability to detect their own heartbeats using a heartbeat counting task (Schandry, 1981). During the heartbeat counting task, participants' heartbeats were monitored via a pulse oximeter (Nonin Model 7500FO) with the sensor mounting attached to their index finger. Participants were required to count their heartbeats during six randomized time windows of varying length $(25,30,35,40,45$, and 60 seconds) and, at the end of each trial, to report the 
number of heartbeats detected to the experimenter. Actual heartbeats were recorded during the interval by the pulse oximeter and transmitted electronically to the data acquisition computer. At the beginning of the heartbeat counting task, participants were given the following verbal instructions: "Without putting your hands on your body, can you count each heartbeat you feel in your body from the time you hear start' to when you hear 'stop'. Count in your head and I will ask you afterwards how many you felt. Some people will feel no heartbeats - that is OK. If that is the case, please tell me none when I ask". Participants were also required to keep their hands on the table in front of them for the duration of the task, to prevent contact with pulse points on the body.

To derive scores for interoceptive accuracy, heartbeat counting scores were calculated on a trial-by-trial basis based upon the ratio of perceived to actual heartbeats: 1-|nbeatsreal nbeatsreported//(nbeatsreal + nbeatsreported)/2 (Garfinkel et al., 2015; Hart, McGowan, Minati, $\&$ Critchley, 2013) and these were averaged to form a mean heartbeat counting score. This measure calculates interoceptive accuracy independent of the number of heartbeats in the trial by normalizing the absolute error in perceived heartbeats as a function of the overall number of heartbeats.

\section{Alexithymia}

Due to time constraints, a sub-sample of children (autistic: $n=30$, non-autistic: $n=49$ ) completed the Alexithymia Questionnaire for Children (AQC, Rieffe, Oosterveld, \& Terwogt, 2006). This scale is based on the original Toronto Alexithymia Scale for adults (TAS-20, Taylor, Ryan, \& Bagby, 1985). The AQC consists of 20 items that represent three factors: 1) Identification subscale - difficulty identifying feelings, 2) Communication subscale - difficulty describing feelings and 3) Externally oriented thinking. The item response format is: 'Not true', 'A bit true', 'True'. Participants could either respond verbally or indicate their response on a chart on the table in front of them. Alexithymia was operationalized as total score on the AQC (Rieffe et al., 2006). Higher scores indicate greater levels of alexithymia. Previously, both Identification and Communication subscales have been found to have good internal consistency (Cronbach's alpha $=0.75$ ), however the External Thinking subscale did not meet criteria for internal consistency (Rieffe et al., 2006). In our samples, the total score was found to have good internal consistency in the non-autistic (Cronbach's alpha $=0.70)$, and autistic participants (Cronbach's alpha $=0.68$ ). Estimates indicated variable internal consistency for the individual 
subscales, ranging from unacceptable to acceptable $(0.45-0.70)$, and scores from these subscales were therefore not entered into analyses.

Procedure

\section{Procedure for participants in the child sample}

Following informed consent, all participants performed the cardiac perception (interoceptive) task, followed by self-report measures. Immediately prior to starting the heartbeat counting task, participants were asked to sit quietly and told to focus internally, to try to feel their heart beating. This was repeated a total of six times using randomized trial lengths $(25,30,35,40,45$, and $60 s)$.

Questionnaires (BPQ, AQC) and the emBODY task were completed by all participants at the time of testing. Younger children and those with more limited language skills had the questions read out to them by the experimenter and indicated their answers using a visual scale. Older participants completed the questionnaires themselves in the presence of the experimenter. IQ assessment was administered to all children using the WASI-II (Wechsler \& Hsiao-pin, 2011). The ADOS-2 (Lord et al., 2012) was administered to all autistic children. The parents of all children were invited to complete the SCQ (Rutter, Bailey, \& Lord, 2003).

\section{Procedure for participants in the adult sample}

Following informed consent, participants completed a booklet containing all measures, including the BPQ, and the emBODY task, which they proceeded to complete in a self-paced manner. Experimenters were available to answer any questions, and participants were fully debriefed upon completion of the measures.

\section{Community engagement}

This research was designed, in part, in response to engagement and discussion with members of the autistic and autism communities. Sensory processing and mental health, particularly affective difficulties, have been outlined as key research goals by community members (Autistica, 2016), and in this study we aim to investigate associations between these two domains. In recruiting our participants, we endeavored to be as inclusive as possible, including modifications for children with more limited expressive language. Community members were 
not, however, directly involved in the design and implementation of the study, or in the analysis and interpretation of the data.

\section{Statistical Analysis}

Statistical analyses were carried out in R Project (R Core Team, 2017). Age, sex and full-scale $I Q$ were entered as covariates in all models, as well as interaction terms between age/IQ and group, due to the significant group difference in sex in our sample, and previous reports of an influence of these variables, including interaction effects, on the measures employed (Hietanen et al., 2016; Mash, Schauder, Cochran, \& Cascio, 2017). Missing data were handled using listwise deletion.

Firstly, for between-group analyses, group differences between autistic and non-autistic children in interoceptive accuracy, interoceptive sensibility, and alexithymia were assessed using multiple linear regression. Secondly, it has been suggested that high rates of alexithymia are responsible for differences observed in interoception in autism (Gaigg, Cornell, \& Bird, 2018; Shah et al., 2016). To this end, we tested whether alexithymia was related to sensations reported on the emBODY task and interoceptive variables, using multiple regression. As in all other analyses, age, sex and IQ were also entered into the models.

Thirdly, in a manipulation check, to determine that the emBODY task produced significant differences in responses across emotion conditions, over and above individual differences in response pattern, a mixed effect model was employed with a fixed effect of condition, and a random intercept for each participant $(n=99)$. A Box-Cox transformation was applied to these activation data prior to analysis to correct for non-normality. The locus of significant differences was further interrogated using post-hoc comparison t-tests; the alpha value was adjusted for multiple comparisons with Bonferroni correction.

Finally, to test the main hypothesis of group differences in emotion differentiation, multiple linear regressions were conducted with emotion differentiation as the dependent variable, with predictors of group, interoceptive accuracy and interoceptive sensibility. Interaction effects between group and interoceptive accuracy and sensibility were also entered into the model. This analysis was performed with emotion differentiation across the whole body entered as the dependent variable. The analysis was then repeated with emotion differentiation in each of the ROls entered as the dependent variable, respectively. Adjusted $R^{2}$ and Predicted $R^{2}$ are 
reported as indications of model fit. Significant interaction effects were explored through posthoc bivariate Pearson correlation analyses; non-parametric Spearman correlations were performed for data that were indicated to be significantly different to the normal distribution by Shapiro-Wilk tests. 


\section{Results}

Between-group analyses

Initial analyses were conducted to test for group differences in interoceptive accuracy, interoceptive sensibility, and alexithymia. We found a significant effect of group for interoceptive sensibility only. Interoceptive sensibility was significantly lower in autistic children than nonautistic children $[B=0.199, t=2.049, p=0.043$ ] (see Table 2 for group-wise summary statistics). Unexpectedly, there was no significant effect of group for either interoceptive accuracy $[B=0.170, t=1.232, p=0.221]$ or alexithymia $[B=-2.239, t=1.596, p=0.115]$.

Table 2: Descriptive statistics for interoceptive accuracy, interoceptive sensibility, alexithymia and emotion differentiation across the whole body and regions of interest. Range, mean and standard deviation in parentheses.

\begin{tabular}{|l|c|c|}
\hline Variable & Autistic (n = 55) & Non-autistic (n = 44) \\
\hline Interoceptive accuracy & $-1.00-0.94,0.22(0.68)$ & $-1.00-0.93,0.34(0.51)$ \\
\hline $\begin{array}{l}\text { Interoceptive sensibility } \\
\text { autistic } \mathbf{n}=49)\end{array}$ & $1.26-3.05,2.25(0.42)$ & $1.33-3.90,2.48(0.49)$ \\
\hline $\begin{array}{l}\text { Whole body emotion } \\
\text { differentiation }\end{array}$ & $0.01-0.46,0.16(0.12)$ & $0.02-0.51,0.16(0.15)$ \\
\hline $\begin{array}{l}\text { Emotion differentiation in } \\
\text { the head }\end{array}$ & $0.00-0.81,0.34(0.18)$ & $0.02-0.87,0.28(0.23)$ \\
\hline $\begin{array}{l}\text { Emotion differentiation in } \\
\text { the heart }\end{array}$ & $0.00-0.63,0.24(0.17)$ & $0.00-0.76,0.21(0.22)$ \\
\hline $\begin{array}{l}\text { Emotion differentiation in } \\
\text { the gut }\end{array}$ & $0.00-0.95,0.30(0.26)$ & $0.00-0.79,0.27(0.23)$ \\
\hline $\begin{array}{l}\text { Emotion differentiation in } \\
\text { the hands }\end{array}$ & $0.04-0.83,0.35(0.20)$ & $0.04-0.78,0.34(0.21)$ \\
\hline
\end{tabular}


Alexithymia was not a significant predictor of emotion differentiation in the body maps, either across the entire sample $[B=0.002, t=1.045, p=0.299]$, or separately, in the autistic [ $B<$ 1.000, $t=-0.064, p=0.950]$ and non-autistic samples $[B=0.003, t=1.105, p=0.275]$. Alexithymia was also not a significant predictor of interoceptive accuracy, at the level of the entire sample $[B=0.024, t=1.998, p=0.050]$, the autistic sample $[B=0.035, t=1.382, p=$ $0.180]$ and the non-autistic sample $[B=0.018, t=1.386, p=0.173]$. The same was true of interoceptive sensibility, across the entire sample $[B=0.016, t=1.709, p=0.092]$, in the autistic sample $[B<1.000, t=1.373, p=0.182]$, and in the non-autistic sample $[B=0.022, t=1.762, p$ $=0.085]$. Across the sample, there was no significant correlation between interoceptive accuracy and interoceptive sensibility $\left[r^{s}(93)=0.258, p=0.804\right]$.

\section{Manipulation check}

Activity on the body maps was found to vary significantly by emotion $(F(1,6)=35.900, p<$ 0.001 ), replicating previous work and indicating that, using the emBODY task, participants produced significantly different maps for the different emotions (Hietanen et al., 2016;

Nummenmaa et al., 2014). Post-hoc comparisons indicated that significant differences were present between anger and calm $(p<0.001)$, anger and disgust $(p<0.001)$, anger and fear $(p<$ $0.025)$, anger and sadness ( $p<0.001)$, calm and disgust $(p<0.001)$, calm and fear $(p<0.001)$, calm and happiness $(p<0.001)$, calm and surprise $(p<0.001)$, disgust and happiness $(p<$ $0.001)$, disgust and sadness $(p<0.001)$, fear and sadness $(p<0.001)$, happiness and sadness $(p<0.001)$, and sadness and surprise $(p<0.001)$.

\section{Bodily emotion differentiation}

Region of interest analysis in the independent adult sample revealed four regions where activity differed significantly between the emotions, specifically in the head, heart, gut and hands (see Supplementary Figure 1).

We conducted multiple regression analyses to test our central hypotheses, that is, that there would be a difference in bodily emotion differentiation between autistic and non-autistic children and adolescents, and that this would be related to interoceptive processing. At the level of the 
whole body, the regression model was significant $\left[F(10,84)=3.482, p<0.001\right.$, Adjusted $\mathrm{R}^{2}=$ 0.209 , Predicted $R^{2}=0.089$ ], but none of the variables of interest had a significant effect on emotion differentiation across the whole body (see Table 2 and Figure 2). The regression models were significant for the head $\left[F(10,84)=3.728, p<0.001\right.$, Adjusted $R^{2}=0.225$, Predicted $\left.\mathrm{R}^{2}=0.124\right]$, heart $\left[F(10,84)=2.847, p=0.004\right.$, Adjusted $\mathrm{R}^{2}=0.164$, Predicted $\mathrm{R}^{2}=$ $0.044]$ and the hands $\left[F(10,84)=2.163, p=0.028\right.$, Adjusted $R^{2}=0.110$, Predicted $\left.\mathrm{R}^{2}=-0.035\right]$. The regression model for the gut did not reach significance $[F(10,84)=1.821, p=0.069$, Adjusted $R^{2}=0.080$, Predicted $\left.R^{2}=-0.042\right]$. Group was a significant predictor of emotion differentiation in the head, heart and hands (see Table 3).

There was a significant group by age interaction effect on emotion differentiation across the whole body, and in each of the ROls (see Figure 2). Post-hoc bivariate correlations revealed that emotion differentiation was significantly correlated with age in non-autistic children, but not in autistic children. This difference was statistically significant when considering emotion differentiation across the whole body (autistic sample $\left[r_{s}(42)=-0.026, p=0.868\right]$, non-autistic sample $\left[r_{s}(53)=-0.558, p<.001\right]$, comparison of slopes $\left.[z=2.890, p=0.004]\right)$, as well as in the head (autistic sample $[r(42)=0.206, p=0.180]$, non-autistic sample $\left[r_{s}(53)=-0.505, p<0.001\right]$, comparison of slopes $[z=3.660, p<0.001]$ ), heart (autistic sample $[r(42)=0.136, p=0.379$, non-autistic sample $\left[r_{s}(53)=-0.389, p=0.003\right]$, comparison of slopes $[z=2.620, p=0.009]$ ), gut (autistic sample $\left[r_{s}(42)=0.094, p=0.546\right]$, non-autistic sample $\left[r_{s}(53)=-0.347, p=0.009\right]$, comparison of slopes $[z=2.180, p=0.029]$ ) and hands (autistic sample $[r(42)=0.079, p=$ $0.610]$, non-autistic sample $\left[r_{s}(53)=-0.488, p<0.001\right]$, comparison of slopes $[z=2.930, p=$ 0.003]).

Table 3: Multiple linear regression models to predict emotion differentiation 


\begin{tabular}{|c|c|c|c|c|}
\hline Region & Effect & $B$ & $t$ & $p$ \\
\hline \multirow{10}{*}{$\begin{array}{l}\frac{\lambda}{0} \\
\frac{8}{0} \\
\frac{0}{0} \\
\frac{1}{3}\end{array}$} & Group & 0.293 & 1.209 & 0.230 \\
\hline & Age & $<0.001$ & 0.104 & 0.917 \\
\hline & Sex & -0.012 & -0.403 & 0.688 \\
\hline & $\mathrm{IQ}$ & 0.002 & 1.784 & 0.078 \\
\hline & Interoceptive accuracy & -0.022 & -0.791 & 0.431 \\
\hline & Interoceptive sensibility & -0.013 & -0.239 & 0.812 \\
\hline & Group $x$ age & -0.027 & -2.836 & 0.006 \\
\hline & Group x IQ & $<0.001$ & -0.606 & 0.546 \\
\hline & Group x interoceptive accuracy & 0.023 & 0.524 & 0.602 \\
\hline & Group x interoceptive sensibility & 0.058 & 0.931 & 0.355 \\
\hline \multirow{10}{*}{$\begin{array}{l}\bar{\nabla} \\
\mathbb{\mathbb { N }} \\
\stackrel{\mathbb{1}}{1}\end{array}$} & Group & 1.264 & 3.316 & 0.001 \\
\hline & Age & 0.014 & 1.108 & 0.271 \\
\hline & Sex & 0.004 & 0.089 & 0.929 \\
\hline & $\mathbf{I Q}$ & 0.005 & 2.844 & 0.006 \\
\hline & Interoceptive accuracy & -0.048 & -1.086 & 0.281 \\
\hline & Interoceptive sensibility & 0.033 & 0.396 & 0.693 \\
\hline & Group x age & -0.052 & -3.439 & $<0.001$ \\
\hline & Group x IQ & -0.006 & -2.316 & 0.023 \\
\hline & Group x interoceptive accuracy & 0.057 & 0.834 & 0.407 \\
\hline & Group x interoceptive sensibility & -0.033 & -0.331 & 0.742 \\
\hline \multirow{6}{*}{$\begin{array}{l}\frac{ \pm}{\mathbb{N}} \\
\stackrel{\mathbb{I}}{I}\end{array}$} & Group & 0.734 & 2.030 & 0.046 \\
\hline & Age & 0.009 & 0.754 & 0.435 \\
\hline & Sex & -0.034 & -0.784 & 0.435 \\
\hline & $\mathrm{IQ}$ & 0.003 & 1.705 & 0.092 \\
\hline & Interoceptive accuracy & -0.023 & -0.558 & 0.578 \\
\hline & Interoceptive sensibility & -0.002 & -0.022 & 0.983 \\
\hline
\end{tabular}




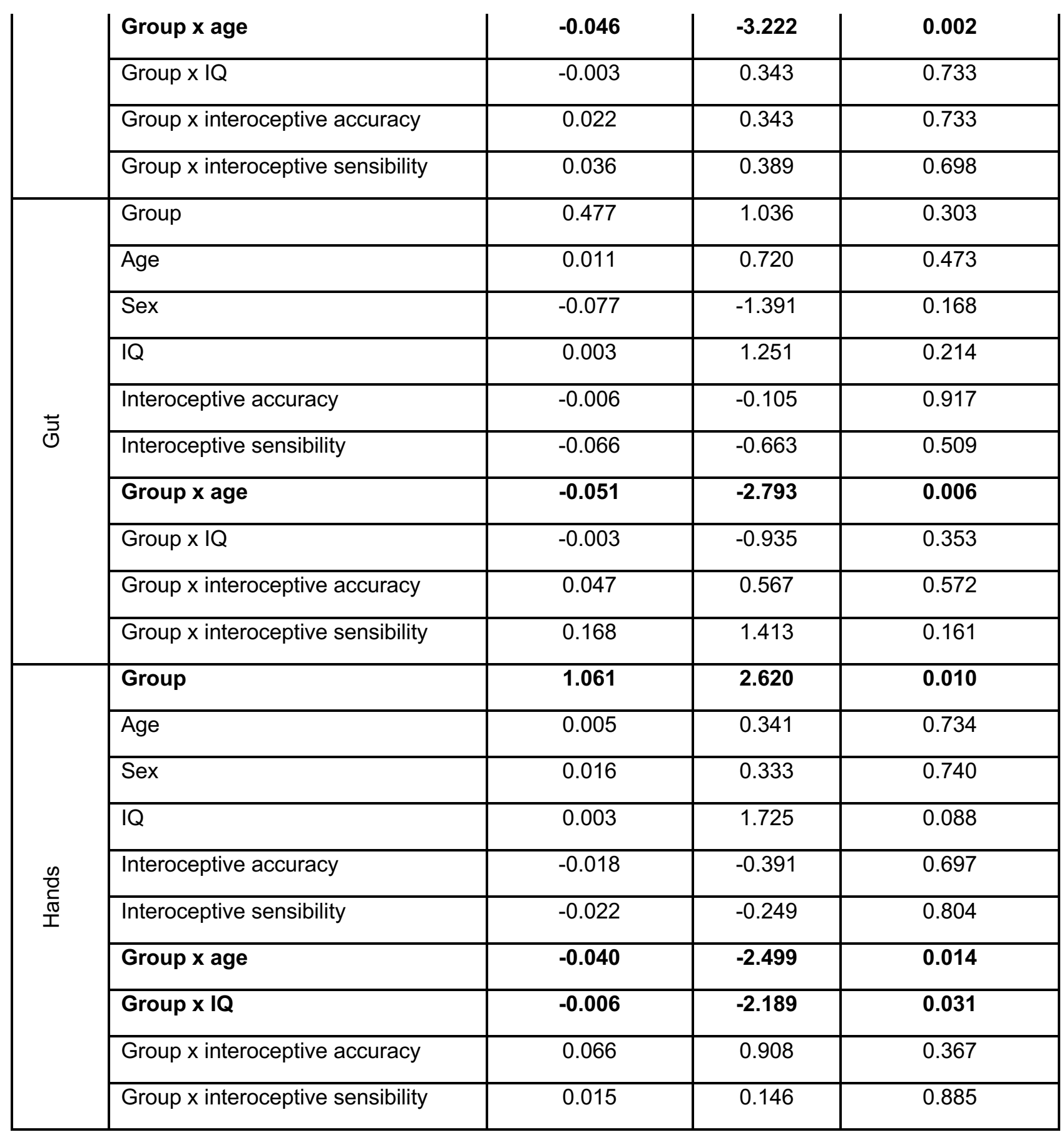

Figure 2: Bodily maps of emotion in autistic and non-autistic children and adolescents. A) Autistic participants reported significantly less differentiation in their bodily experiences of emotion than non-autistic participants in several emotion-associated ROls. That is, there was, on average, more similarity between different emotions for autistic participants in the head, the heart and the hands. The color-bar reflects mean activity. Red through yellow indicates 
increasing bodily activity, while dark through light blue indicates decreasing bodily activity. B) Non-autistic children showed reduced bodily emotion differentiation with age; this developmental pattern was not seen in autistic children. 

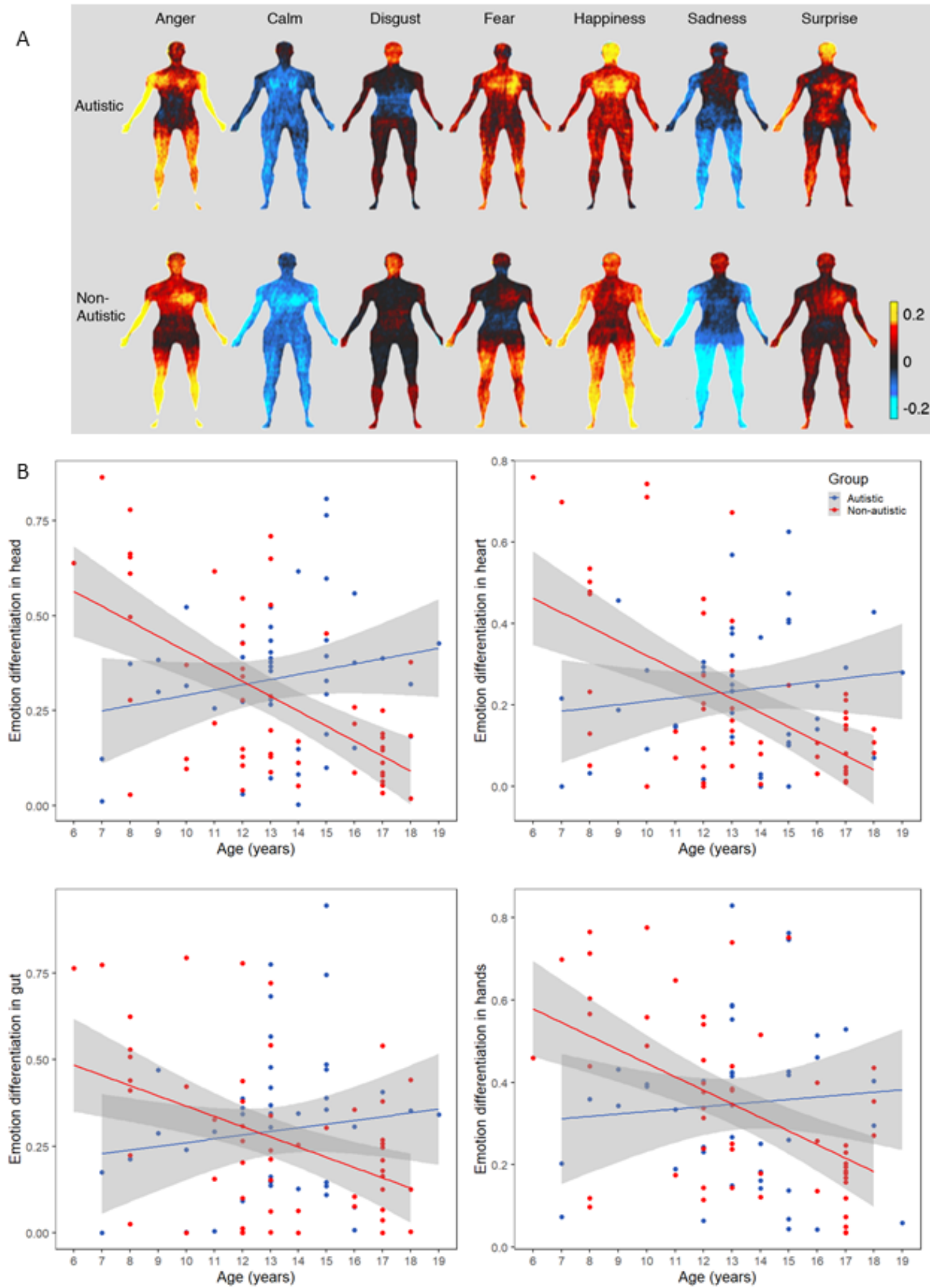


\section{Discussion}

In this study, we tested the hypothesis that there would be significant differences in how autistic and non-autistic children report experiencing emotions in their bodies. Furthermore, we predicted that the bodily experience of emotion would be positively related to interoceptive accuracy but negatively related to interoceptive sensibility. Our results supported the first hypothesis, revealing reduced bodily emotion differentiation in autistic children in several regions, namely the head, the heart and the hands. The activity of these regions had been identified in a large independent, adult sample as dissociating between the different emotional experiences tested. The only region where differences were not seen in the autistic participants was the gut. The absence of significant differences in differentiation at the level of the whole body suggests these results are not due to more generalized differences in the variability of responses across conditions in the autistic sample, and suggest the results are specific to emotion-related regions of the body.

There was no evidence, however, for the involvement of either subjective or objective interoceptive processing in the bodily representation of emotion in this sample. Emotions are multi-component and comprise cognitive, motor, subjective and physiological changes (Etkin, Büchel, \& Gross, 2015). Activity reported in the bodily maps may reflect many or all of these components, not just the interoceptive detection of physiological changes. Developmentally, there may be (at least) two routes to the acquisition of socio-emotional understanding (Pezzulo et al., 2016). This argument has previously been made in the context of explicit and implicit theory of mind (Heyes \& Frith, 2014). In this review, the authors proposed that neurotypical infants are born with some automatic or implicit neurocognitive means of predicting others' behavior, but an explicit ability to deliberate and talk about mental states develops through the mechanisms of cultural inheritance. If we are to apply this framework to the ability to understand emotional states, we can suppose that one route to emotional competency involves incidental learning about the contingencies between interoceptive and affective states. The other route, however, may be scaffolded by overt learning processes, such as conversation and direct instruction. In the present study, there was no evidence of a significant contribution of either objective or subjective interoceptive processing, to emotion differentiation - in autistic or nonautistic children. Measures than quantify participants' ability to interpret interoceptive signals in 
terms of their emotional significance may have a more proximal association with emotion differentiation, than the detection of interoceptive signals per se.

Here, we replicated previous reports of reduced emotion differentiation in autism (Erbas et al., 2013; Rieffe et al., 2007), using a novel embodied approach, previously unapplied to this population. Reduced emotion differentiation in autism may be related to the affective challenges that autistic people are more likely to experience, including anxiety and depression (Gadow et al., 2004; Kim et al., 2000; Muris et al., 1998; Simon of et al., 2008; Sukhodolsky et al., 2008; Weisbrot et al., 2005). More differentiated emotional experiences are associated with a greater capacity to regulate emotion, particularly negative emotion (Barrett et al., 2001), and less frequent use of maladaptive coping strategies, such as self-injurious behavior (Zaki et al., 2013). It is suggested that high emotion differentiation abilities permit an individual to distance themselves from distressing bodily sensations and feelings (Kashdan, Barrett, \& McKnight, 2015). Indeed, previous work in adults has shown that alexithymia mediates the relationship between interoceptive processing and anxiety (Palser, Palmer et al., 2018). Within the general population, those with both the highest rates of interoceptive sensibility and high levels of alexithymia, were most at risk of clinically-significant anxiety symptoms. Future cross-sectional work should explore the relationship between performance on this task and the presence of cooccurring psychiatric conditions, and longitudinal work should examine its utility as a predictor of later difficulties.

In the present study, decreasing emotion differentiation with age was observed in non-autistic children. This goes against an existing body of research, which suggests that emotion differentiation should develop linearly throughout childhood and adolescence, until reaching adult levels (Hietanen et al., 2016; Widen, 2013). While young children tend only to differentiate emotions into the two categories of positive and negative (Larsen, To, \& Fireman, 2007), this differentiation is thought to increase as children learn about the different categories of positive and negative emotion. More recent research, however, suggests an alternative view: that emotion differentiation might actually follow a U-shaped trajectory from early childhood to adulthood, with the lowest point of the curve occurring during adolescence (Nook, Sasse, Lambert, McLaughlin, \& Somerville, 2018). On this view, children only begin to understand that more than one emotion can be experienced at the same time around the age of nine years; prior to this age, emotions are viewed as highly discrete, differentiated experiences, precisely because they cannot co-occur (Harter \& Buddin, 1987; Wintre \& Vallence, 1994; Larsen et al., 2007). As this understanding increases, and adolescents shift away from the belief that 
emotions are mutually exclusive, emotions become less differentiated. An increase in emotion differentiation only occurs as adolescents move into adulthood, a time when emotion concepts become more defined, and the experience of different emotions can be delineated despite cooccurring (Nook et al., 2018). The present results seem to support this second hypothesis, showing decreasing variance in emotion-related bodily regions between the ages of six and 19 years in neurotypical development. In autistic participants, we found no effect of age on emotion differentiation. As such, decreasing emotion differentiation throughout childhood may be an adaptive trend, one that is delayed or relatively absent in autistic children and adolescents.

Emotion differentiation is most typically measured using language-based self-report measures, where participants repeatedly report on their emotional experiences, and the number of distinct emotion terms used is quantified. It has been suggested that reduced emotion differentiation in autism may be related to an inability to understand and use emotion words (Erbas et al., 2013). Here, however, we show that using a non-linguistic measure, autistic children continue to show differences in emotion differentiation. Participants needed only to recognize the emotion word for the trial. That said, it is possible that language is more fundamentally involved in emotion differentiation, wherein learnt emotion terminology is used to categorize incoming sensations, over time contributing to a richer emotional internal world. In line with this possibility, some have argued that those with more limited emotion knowledge, in the form of a reduced emotional vocabulary, will conceptualize sensory inputs in a less differentiated fashion (Kashdan et al., 2015). Similarly, although previous reports have suggested no difference in the resultant bodily maps between memory-based and emotion induction paradigms (Nummenmaa et al., 2014), these studies were conducted with neurotypical individuals. Alterations in episodic memory have previously been found in autism (Crane \& Goddard, 2008; Lind \& Bowler, 2010). It is possible, then, that group differences represent shallower encoding of emotionally salient events, which has down-stream effects on the ability to report the bodily sensations associated with these states when prompted.

As we did not find an association with interoceptive processing, it is possible that more language-based and memory pathways contributed to the reduced emotion differentiation observed in the autistic children and adolescents studied here. Further work will be needed to tease this apart, but if this is the case, interventions designed to increase children's emotion word knowledge or encoding of emotionally salient events may prove fruitful in increasing emotion differentiation in this population, with important effects on reducing the risk of affective difficulties. 
We should consider methodological explanations for the absence of interoceptive contributions in the results observed. There has been increasing discussion of the limitations of the heartbeat counting task as a measure of interoceptive accuracy. There are several studies now reporting that a participant's beliefs and knowledge about their heart rate can influence performance on this task (Ehlers et al., 2000; Murphy et al., 2018; Ring \& Brener, 1996), suggesting that it may not be a particularly good measure of an individual's access to interoceptive signals. Indeed, in the present study we did not replicate previous findings of altered interoceptive processing in autism (Garfinkel et al., 2016; Palser, Fotopoulou, et al., 2018; Mul et al., 2018). While the group means were in the expected direction, the difference was not significant. Interoceptive differences are unlikely to be present in all autistic people (see, for example, studies by Nicholson et al., 2018; Shah et al., 2016; \& Schauder et al., 2015), and the ability to detect these differences is reduced by an insensitive measure and extraneous participant characteristics, including body mass (Montgomery \& Jones, 1984), blood pressure (O'Brien, Reid, \& Jones, 1998), and working memory capacity. Replication studies with large samples and reliable methods are needed to ensure sufficient power to detect associations with diagnostic status in the presence of individual differences.

Surprisingly, considering previous research findings (Griffin et al., 2016; Hill et al., 2004), we did not find higher rates of alexithymia in the autistic children and adolescents in the current sample and found no association with bodily emotion differentiation. As demonstrated in the current sample, the validity and reliability of these measures in children, particularly those diagnosed with autism, is still uncertain, with a lack of association between child- and parent-reported alexithymia observed (Griffin et al., 2016). Conceptually, emotion differentiation and alexithymia have been considered different measures of the same construct, and thus assumed to be interrelated. However, there are previous findings of a lack of association (Edwards \& Wupperman, 2016), suggesting these may be separate and distinct. Some authors have argued that alexithymia represents a disruption in low-level emotion processing, while differentiation of subjective emotional states is a higher-order process (Nemiah, 1977; Sifneos, 1994). As such, alexithymia may reflect a disruption in the detection of interoceptive signals (Brewer, Cook, \& Bird, 2016; Herbert, Herbert, \& Pollatos, 2011; Shah et al., 2016), while emotion differentiation involves language and the capacity to reason about mental states.

In conclusion, the present findings suggest the bodily experience of emotion is less differentiated in autistic children and adolescents, which may have knock-on effects on emotion regulation difficulties and anxiety. No evidence was found, however, to suggest that these 
differences relate to either subjective or objective interoceptive processing. Caution is warranted given the apparent limitations of current measures of interoception. Nevertheless, these findings suggest that interoception may not be the only source of differences in emotional understanding.

\section{Funding}

This work was supported by a PhD studentship awarded to E. Palser from the Economic and Social Research Council (ESRC) and the Medical Research Council (MRC) (ES/J500185/1). E. Pellicano was supported by a grant from the MRC (MR/J013145/1) and Philip Leverhulme Prize awarded by the Leverhulme Trust. A. Fotopoulou was supported by a 'European Research Council Starting Investigator Award' [ERC-2012-STG GA313755]. 


\section{References}

American Psychiatric Association. (2000). Diagnostic and Statistical Manual of Mental Disorders. 4th Edition. Text Revision (DSM-IV-TR). Washington, DC: American Psychiatric Association.

Autistica. (2016). Your questions: Shaping autism research [PDF file]. Retrieved June 16, 2020, from https://www.autistica.org.uk/downloads/files/Autism-Top-10-Your-Priorities-forAutism-Research.pdf.

Barrett, L. F., Gross, J., Christensen, T. C., \& Benvenuto, M. (2001). Knowing what you're feeling and knowing what to do about it: Mapping the relation between emotion differentiation and emotion regulation. Cognition \& Emotion, 15(6), 713-724.

Barrett, L. F., Quigley, K. S., Bliss-Moreau, E., \& Aronson, K. R. (2004). Interoceptive sensitivity and self-reports of emotional experience. JPSP, 87, 684-697.

Brewer, R., Cook, R., \& Bird, G. (2016). Alexithymia: a general deficit of interoception. Royal Society Open Science, 3(10), 150664.

Crane, L., \& Goddard, L. (2008). Episodic and semantic autobiographical memory in adults with autism spectrum disorders. Journal of Autism and Developmental Disorders, 38(3), 498506.

Damasio, A. R. (1994). Descartes' error and the future of human life. Scientific American, 271(4), 144.

Edwards, E. R., \& Wupperman, P. (2017). Emotion regulation mediates effects of alexithymia and emotion differentiation on impulsive aggressive behavior. Deviant Behavior, 38(10), 1160-1171.

Ehlers, A., Mayou, R. A., Sprigings, D. C., \& Birkhead, J. (2000). Psychological and perceptual factors associated with arrhythmias and benign palpitations. Psychosomatic Medicine, 62(5), 693-702.

Etkin, A., Büchel, C., \& Gross, J. J. (2015). The neural bases of emotion regulation. Nature Reviews Neuroscience, 16(11), 693-700.

Erbas, Y., Ceulemans, E., Boonen, J., Noens, I., \& Kuppens, P. (2013). Emotion differentiation in autism spectrum disorder. Research in Autism Spectrum Disorders, 7(10), 1221-1227.

Ernst, J., Böker, H., Hättenschwiler, J., Schüpbach, D., Northoff, G., Seifritz, E., \& Grimm, S. (2013). The association of interoceptive awareness and alexithymia with neurotransmitter concentrations in insula and anterior cingulate. Social Cognitive and Affective Neuroscience, 9(6), 857-863.

Gadow, K. D., DeVincent, C. J., Pomeroy, J., \& Azizian, A. (2004). Psychiatric symptoms in preschool children with PDD and clinic and comparison samples. Journal of Autism and Developmental Disorders, 34(4), 379-393.

Gaigg, S. B., Cornell, A. S., \& Bird, G. (2018). The psychophysiological mechanisms of alexithymia in autism spectrum disorder. Autism, 22(2), 227-231. 
Garfinkel, S. N., \& Critchley, H. D. (2013). Interoception, emotion and brain: new insights link internal physiology to social behaviour. Commentary on: "Anterior insular cortex mediates bodily sensibility and social anxiety" by Terasawa et al.(2012). Social Cognitive and Affective Neuroscience, 8(3), 231-234.

Garfinkel, S. N., Seth, A. K., Barrett, A. B., Suzuki, K., \& Critchley, H. D. (2015). Knowing your own heart: distinguishing interoceptive accuracy from interoceptive awareness. Biological Psychology, 104, 65-74.

Garfinkel, S. N., Tiley, C., O'Keeffe, S., Harrison, N. A., Seth, A. K., \& Critchley, H. D. (2016). Discrepancies between dimensions of interoception in autism: implications for emotion and anxiety. Biological Psychology, 114, 117-126.

Gotham, K., Pickles, A., \& Lord, C. (2009). Standardizing ADOS scores for a measure of severity in autism spectrum disorders. Journal of Autism and Developmental Disorders, 39(5), 693-705.

Gotham, K., Risi, S., Pickles, A., \& Lord, C. (2007). The Autism Diagnostic Observation Schedule: revised algorithms for improved diagnostic validity. Journal of Autism and Developmental Disorders, 37(4), 613.

Griffin, C., Lombardo, M. V., \& Auyeung, B. (2016). Alexithymia in children with and without autism spectrum disorders. Autism Research, 9(7), 773-780.

Hart, N., McGowan, J., Minati, L., \& Critchley, H. D. (2013). Emotional regulation and bodily sensation: interoceptive awareness is intact in borderline personality disorder. Journal of Personality Disorders, 27(4), 506.

Herbert, B. M., Herbert, C., \& Pollatos, O. (2011). On the relationship between interoceptive awareness and alexithymia: is interoceptive awareness related to emotional awareness?. Journal of Personality, 79(5), 1149-1175.

Heyes, C., \& Frith, C. D. (2014). The cultural evolution of mind reading. Science, 344(6190), 1243091.

Hietanen, J. K., Glerean, E., Hari, R., \& Nummenmaa, L. (2016). Bodily maps of emotions across child development. Developmental Science, 19(6), 1111-1118.

Hill, E., Berthoz, S., \& Frith, U. (2004). Brief report: Cognitive processing of own emotions in individuals with autistic spectrum disorder and in their relatives. Journal of Autism and Developmental Disorders, 34(2), 229-235.

James, W. (1884). What is an emotion? Mind, 9(34), 188-205.

Jung, W. M., Ryu, Y., Lee, Y. S., Wallraven, C., \& Chae, Y. (2017). Role of interoceptive accuracy in topographical changes in emotion-induced bodily sensations. PloS one, 12(9), e0183211.

Kanner, L. (1943). Autistic disturbances of affective contact. Nervous Child, 2(3), 217-250.

Kashdan, T. B., Barrett, L. F., \& McKnight, P. E. (2015). Unpacking emotion differentiation: Transforming unpleasant experience by perceiving distinctions in negativity. Current Directions in Psychological Science, 24(1), 10-16. 
Kashdan, T. B., \& Farmer, A. S. (2014). Differentiating emotions across contexts: Comparing adults with and without social anxiety disorder using random, social interaction, and daily experience sampling. Emotion, 14(3), 629.

Kim, J. A., Szatmari, P., Bryson, S. E., Streiner, D. L., \& Wilson, F. J. (2000). The prevalence of anxiety and mood problems among children with autism and Asperger syndrome. Autism, 4(2), 117-132.

Larsen, J. T., To, Y. M., \& Fireman, G. (2007). Children's understanding and experience of mixed emotions. Psychological Science, 18, 186-191.

Levenson, R. W. (2003). Blood, sweat, and fears: The autonomic architecture of emotion. Annals of the New York Academy of Sciences, 1000(1), 348-366.

Liberatos, P., Link, B. G., \& Kelsey, J. L. (1988). The measurement of social class in epidemiology. Epidemiologic Reviews, 10(1), 87-121.

Lind, S. E., \& Bowler, D. M. (2010). Episodic memory and episodic future thinking in adults with autism. Journal of Abnormal Psychology, 119(4), 896.

Longarzo, M., D'Olimpio, F., Chiavazzo, A., Santangelo, G., Trojano, L., \& Grossi, D. (2015). The relationships between interoception and alexithymic trait. The Self-Awareness Questionnaire in healthy subjects. Frontiers in Psychology, 6, 1149.

Lord, C., Rutter, M., DiLavore, P. C., Risi, S., Gotham., K., Bishop, S. L., ... \& Guthrie, W. (2012). ADOS-2: Autism diagnostic observation schedule, second edition. Boston, MA: Hogrefe.

Mash, L. E., Schauder, K. B., Cochran, C., Park, S., \& Cascio, C. J. (2017). Associations between interoceptive cognition and age in autism spectrum disorder and typical development. Journal of Cognitive Education and Psychology, 16(1), 23-37.

Montgomery, W. A., \& Jones, G. E. (1984). Laterality, emotionality, and heartbeat perception. Psychophysiology, 21(4), 459-465.

Mul, C. L., Stagg, S. D., Herbelin, B., \& Aspell, J. E. (2018). The feeling of me feeling for you: Interoception, alexithymia and empathy in autism. Journal of Autism and Developmental Disorders, 1-15.

Muris, P., Steerneman, P., Merckelbach, H., Holdrinet, I., \& Meesters, C. (1998). Comorbid anxiety symptoms in children with pervasive developmental disorders. Journal of Anxiety Disorders, 12(4), 387-393.

Murphy, J., Millgate, E., Geary, H., Ichijo, E., Coll, M-P., Brewer, R., Catmur, C., \& Bird, G. (2018). Knowledge of resting heart rate mediates the relationship between intelligence and the heartbeat counting task. Biological Psychology, 133, 1-3.

Nicholson, T., Williams, D. M., Grainger, C., Christensen, J. F., Calvo-Merino, B., \& Gaigg, S. B. (2018). Interoceptive impairments do not lie at the heart of Autism or Alexithymia. Journal of Abnormal Psychology, 127(6), 612-622.

Nemiah, J. C. (1977). Alexithymia: theoretical considerations. Psychotherapy and Psychosomatics, 28(1/4), 199-206. 
Nook, E. C., Sasse, S. F., Lambert, H. K., McLaughlin, K. A., \& Somerville, L. H. (2018). The nonlinear development of emotion differentiation: Granular emotional experience is low in adolescence. Psychological Science, 29(8), 1346-1357.

Nummenmaa, L., Glerean, E., Hari, R., \& Hietanen, J. K. (2014). Bodily maps of emotions. Proceedings of the National Academy of Sciences, 111(2), 646-651.

O'Brien, W. H., Reid, G. J., \& Jones, K. R. (1998). Differences in heartbeat awareness among males with higher and lower levels of systolic blood pressure. International Journal of Psychophysiology, 29(1), 53-63.

Palser, E. R., Fotopoulou, A., Pellicano, E., \& Kilner, J. M. (2018). The link between interoceptive processing and anxiety in children diagnosed with autism spectrum disorder: Extending adult findings into a developmental sample. Biological Psychology, $136,13-21$.

Palser, E. R., Fotopoulou, A., Pellicano, E., \& Kilner, J. M. (2019). Dissociation in how core autism features relate to interoceptive dimensions: evidence from cardiac awareness in children. Journal of Autism and Developmental Disorders, 1-11.

Palser, E. R., Palmer, C. E., Galvez-Pol, A., Hannah, R., Fotopoulou, A., \& Kilner, J. M. (2018). Alexithymia mediates the relationship between interoceptive sensibility and anxiety. PloS one, 13(9), e0203212.

Pezzulo, G., Vosgerau, G., Frith, U., Hamilton, A. F. de C., Heyes, C., Iriki, A., Jörntell, H., König, P., Nagal., S. K., Oudeyer, P-Y., Rupert, R. D., \& Tramacere, A. (2016). Acting up: An approach to the study of cognitive development. In A. K. Engel, K. J. Friston, \& D. Kragic (Eds.), The pragmatic turn: Toward action-oriented views of cognitive science (pp. 49-80). Cambridge, MA: MIT Press.

Pollatos, O., Herbert, B. M., Matthias, E., \& Schandry, R. (2007). Heart rate after emotional picture presentation is modulated by interoceptive awareness. International Journal of Psychophysiology, 63, 117-124.

Pond Jr, R. S., Kashdan, T. B., DeWall, C. N., Savostyanova, A., Lambert, N. M., \& Fincham, F. D. (2012). Emotion differentiation moderates aggressive tendencies in angry people: $A$ daily diary analysis. Emotion, 12(2), 326.

Porges, S. (1993). Body perception questionnaire. Laboratory of Developmental Assessment, University of Maryland.

Rieffe, C., Oosterveld, P., \& Terwogt, M. M. (2006). An alexithymia questionnaire for children: Factorial and concurrent validation results. Personality and Individual Differences, 40(1), 123-133.

Rieffe, C., Terwogt, M. M., \& Kotronopoulou, K. (2007). Awareness of single and multiple emotions in high-functioning children with autism. Journal of Autism and Developmental Disorders, 37(3), 455-465.

Ring, C., \& Brener, J. (1996). Influence of beliefs about heart rate and actual heart rate on heartbeat counting. Psychophysiology, 33(5), 541-546. 
Rutter, M., Bailey, A., \& Lord, C. (2003). The social communication questionnaire: Manual. Western Psychological Services.

Rutter, M., Le Couteur, A., \& Lord, C. (2003). Autism diagnostic interview-revised. Los Angeles, CA: Western Psychological Services, 29, 30.

Samson, A. C., Hardan, A. Y., Podell, R. W., Phillips, J. M., \& Gross, J. J. (2015). Emotion regulation in children and adolescents with autism spectrum disorder. Autism Research, 8(1), 9-18.

Schandry, R. (1981). Heart beat perception and emotional experience. Psychophysiology, 18(4), 483-488.

Schauder, K. B., Mash, L. E., Bryant, L. K., \& Cascio, C. J. (2015). Interoceptive ability and body awareness in autism spectrum disorder. Journal of Experimental Child Psychology, 131, 193-200.

Shah, P., Hall, R., Catmur, C., \& Bird, G. (2016). Alexithymia, not autism, is associated with impaired interoception. Cortex, 81, 215-220.

Sifneos, P. E. (1994). Affect deficit and alexithymia. New Trends in Experimental \& Clinical Psychiatry.

Simonoff, E., Pickles, A., Charman, T., Chandler, S., Loucas, T., \& Baird, G. (2008). Psychiatric disorders in children with autism spectrum disorders: prevalence, comorbidity, and associated factors in a population-derived sample. Journal of the American Academy of Child \& Adolescent Psychiatry, 47(8), 921-929.

Soke, G. N., Rosenberg, S. A., Hamman, R. F., Fingerlin, T., Robinson, C., Carpenter, L., Giarelli, E., Lee, L. C., Wiggins, L. D., Durkin, M. S., \& DiGuiseppi, C. (2016). Brief report: prevalence of self-injurious behaviors among children with autism spectrum disorder-a population-based study. Journal of autism and developmental disorders, 46(11), 3607-3614.

Steptoe, A., \& Vögele, C. (1992). Individual differences in the perception of bodily sensations: the role of trait anxiety and coping style. Behaviour Research and Therapy, 30(6), 597607.

Sukhodolsky, D. G., Scahill, L., Gadow, K. D., Arnold, L. E., Aman, M. G., McDougle, C. J., McCracken, J. T., Tierney, E., White, S. W., Lecavalier, L., \& Vitiello, B. (2008). Parentrated anxiety symptoms in children with pervasive developmental disorders: Frequency and association with core autism symptoms and cognitive functioning. Journal of Abnormal Child Psychology, 36(1), 117-128.

Taylor, G. J., Ryan, D. P. \& Bagby, R. M. (1985). Toward the development of a new self-report alexithymia scale. Psychotherapy and Psychosomatics, 44, 191-199.

Wechsler, D., \& Hsiao-pin, C. (2011). WASI II: Wechsler Abbreviated Scale of Intelligence. (2 ${ }^{\text {nd }}$ ed.) San Antonio, TX: Psychological Corporation. 
Weisbrot, D. M., Gadow, K. D., DeVincent, C. J., \& Pomeroy, J. (2005). The presentation of anxiety in children with pervasive developmental disorders. Journal of Child \& Adolescent Psychopharmacology, 15(3), 477-496.

Werling, D. M., \& Geschwind, D. H. (2013). Sex differences in autism spectrum disorders. Current Opinion in Neurology, 26(2), 146.

Werner, N. S., Jung, K., Duschek, S., \& Schandry, R. (2009). Enhanced cardiac perception is associated with benefits in decision-making. Psychophysiology, 46(6), 1123-1129.

Werner, N. S., Kerschreiter, R., Kindermann, N. K., \& Duschek, S. (2013). Interoceptive awareness as a moderator of affective responses to social exclusion. Journal of Psychophysiology, 27, 39-50.

Widen, S. C. (2013). Children's interpretation of facial expressions: The long path from valencebased to specific discrete categories. Emotion Review, 5, 72-77.

Widen, S. C., \& Russell, J. A. (2010). Differentiation in preschooler's categories of emotion. Emotion, 10(5), 651.

Wiens, S., Mezzacappa, E., \& Katkin, E. (2000). Heartbeat detection and the experience of emotions. Cognition and Emotion, 14, 417-427.

World Health Organisation (WHO) (1993). International Classification of Disease ICD-10. Geneva: WHO.

Zaki, L. F., Coifman, K. G., Rafaeli, E., Berenson, K. R., \& Downey, G. (2013). Emotion differentiation as a protective factor against nonsuicidal self-injury in borderline personality disorder. Behavior Therapy, 44(3), 529-540.

Zaki, J., Davis, J. I., \& Ochsner, K. N. (2012). Overlapping activity in anterior insula during interoception and emotional experience. Neuroimage, 62(1), 493-499 